\title{
Saliency maps for finding changes in visual scenes?
}

\author{
Heinrich René Liesefeld ${ }^{1}$ Anna Marie Liesefeld ${ }^{1}$ Hermann J. Müller ${ }^{1,2}$ • \\ Dragan Rangelov ${ }^{1,3}$
}

Published online: 17 July 2017

(C) The Psychonomic Society, Inc. 2017

\begin{abstract}
Sudden changes in the environment reliably summon attention. This rapid change detection appears to operate in a similar fashion as pop-out in visual search, the phenomenon that very salient stimuli are directly attended, independently of the number of distracting objects. Pop-out is usually explained by the workings of saliency maps, i.e., map-like representations that code for the conspicuity at each location of the visual field. While past research emphasized similarities between pop-out search and change detection, our study highlights differences between the saliency computations in the two tasks: in contrast to pop-out search, saliency computation in change detection (i) operates independently across different stimulus properties (e.g., color and orientation), and (ii) is little influenced by trial history. These deviations from pop-out search are not due to idiosyncrasies of the stimuli or task design, as evidenced by a replication of standard findings in a comparable visual-search design. To explain these results, we outline a model of change detection involving the computation of feature-difference maps, which explains the known similarities and differences with visual search.
\end{abstract}

Heinrich René Liesefeld

Heinrich.Liesefeld@psy.lmu.de

$\triangle$ Dragan Rangelov

d.rangelov@uq.edu.au

1 Department of Psychology, Ludwig-Maximilians-Universität, Munich, Germany

2 Department of Psychology, Birkbeck College, London, UK

3 Queensland Brain Institute, The University of Queensland, Brisbane, QLD, Australia
Keywords Visual short-term memory $\cdot$ Race-model inequality (RMI) · Priority map · Co-activation .

Intertrial-sequence effects

Perception provides only a sparse and biased representation of the external world. It is clearly selective, with preference for the most salient stimuli. Additionally, our current and recent motives and action goals influence what we select (Awh, Belopolsky, \& Theeuwes, 2012), with a critical role assigned to memory processes (e.g., Kuhl \& Chun, 2014). In the present study, we examine a newly discovered influence of memory on perception (Hyun, Woodman, Vogel, \& Luck, 2009) and discuss its underlying mechanisms.

Many studies have shown that information held in visual working memory (VWM) influences perception (e.g., Downing, 2000; Olivers, Meijer, \& Theeuwes, 2006; Soto, Heinke, Humphreys, \& Blanco, 2005; Reinhart, McClenahan, \& Woodman, 2016). Since even a taskirrelevant stimulus matching VWM content is likely to capture attention, it has been suggested that VWM content may serve as an attentional template influencing stimulus selection (for reviews, see Olivers, Peters, Houtkamp, \& Roelfsema, 2011; Soto, Hodsoll, Rotshtein, \& Humphreys, 2008). To date, this attentional-template hypothesis is the dominant theoretical account for interactions between VWM and perception (Desimone \& Duncan, 1995; Duncan \& Humphreys, 1989; Humphreys \& Müller, 1993; Olivers et al., 2011; Soto et al., 2008; Shomstein \& Yantis, 2002).

Recently, however, Hyun et al. (2009) reported a VWM effect on spatial attention that cannot be explained by attentional templates. They used a change-detection task, in which participants had to discern a change between two stimulus displays presented consecutively with some delay. Each display comprised several differently oriented bars that, on a 
portion of trials, were identical across displays. On the remaining trials, one bar changed its orientation. Analysis of eventrelated potentials (ERPs) locked to the second display revealed a negative deflection contralateral to the change location in the 170- to 270-ms range. This lateralized ERP component is referred to as $\mathrm{N} 2 \mathrm{pc}$ (negativity in the $\mathrm{N} 2$ range over posterior electrode sites contralateral to the eliciting object), and the consensus in the literature is that the N2pc reflects dynamics of spatial attention (for a review, see Luck, 2012). Consequently, the results of Hyun et al. imply that the change attracts spatial attention. Importantly, the latency of the N2pc was completely independent of the numbers of elements, indicating that attention was guided directly to the change location. Since the precise feature values of the changed elements are unpredictable, participants were effectively prevented from adopting any particular attentional template that could have mediated this guidance. Accordingly, some other kind of signal must have guided attention.

Guidance of attention towards the change location bears a striking resemblance with one of the most robust and theoretically interesting findings in visual-attention research. When participants search for one element that strongly differs from surrounding homogeneous distractors (e.g., a tilted bar amongst many vertical bars), response speed varies little with an increase in the number of distractors (e.g., Liesefeld, Moran, Müller, \& Zehetleitner, 2016; Nothdurft, 1993). Such flat search slopes for feature singletons are considered a hallmark of pop-out search, in which the target element is mandatorily selected first.

Most theories of visuo-spatial attention account for pop-out in terms of an early (pre-attentive) segmentation mechanism that partitions the scene into regions of high and low interest: a saliency map. This map codes the saliency at each location of visual input, with the most salient location summoning focal attention (e.g., Wolfe, 2007). Saliency depends on local feature contrasts; thus, for example, a strongly tilted bar surrounded by vertical bars would yield a strong featurecontrast signal and pop out. With this in mind, Hyun et al.'s (2009) findings may be taken to indicate that, similar to feature singletons, changes pop out because their locations are assigned the highest values on some saliency map. Importantly, this map would not represent feature contrasts but rather change contrasts, that is, differences between past (maintained in VWM) and present (currently displayed) sensory input. The present study was designed to investigate the extent to which similar saliency-computation mechanisms are involved in finding visual targets and in detecting changes, by testing whether behavioral effects that are robustly observed in pop-out search tasks persist in the change-detection task.

A robust effect typically attributed to the dynamics of saliency maps are redundancy gains: A search target differing from distractors in two attributes (redundant target) is found faster than a target differing in just one attribute (single target); for example, a red-and-tilted target against green-vertical distractors is easier to find than a red-vertical target. As previously demonstrated (e.g., Miller, 1982), redundancy gains can arise from a simple race between two signals. Evidence for the presence of the two target attributes is accumulated in parallel and the first accumulator that reaches some criterion boundary will trigger target detection. As both accumulators can trigger target detection, the one that reaches the boundary first determines the response time on a given trial. If sometimes one and sometimes the other signal wins the race (if the distributions of detection times for the two single signals are overlapping), this yields faster detection of redundant signals relative to even the detection of the, on average, easier single signal (redundancy gains).

Race models can explain redundancy gains only up to a certain boundary: if the race model holds, response times (RTs) in the redundant condition can never be faster than as predicted by an ideal race between the single signals. Violations of this so-called race-model inequality $(R M I)$ have been interpreted as evidence for co-activation, which means that the two signals are combined into one stronger signal, rather than simply racing against each other (e.g., Feintuch \& Cohen, 2002; Krummenacher, Müller, \& Heller, 2001, 2002a, b; Krummenacher \& Müller, 2014; Mordkoff \& Yantis, 1993). In the context of visual search, RMI violations imply the existence of a supra-dimensional (master) saliency map at which all feature contrasts (e.g., both color and orientation) are pooled together. Different attributes of the same item (e.g., a redundant color/orientation target) would coactivate the same location on the spatio-topically organized master map, thereby jointly guiding spatial attention (e.g., Töllner, Zehetleitner, Krummenacher, \& Müller, 2011). As the combined signal is stronger than each of the single signals, target detection is expedited, resulting in strong redundancy gains.

As the RMI constitutes the upper bound on race models, RMI violations are a conservative criterion for co-activation (Miller, 1982); that is, the presence of RMI violations provides strong evidence for co-activation. The absence of RMI violations, by contrast, does not constitute strong evidence against co-activation (see Ratko-Dehnert, 2013, for an in-depth simulation study directly assessing this lack of power of the RMIviolation test). For the visual domain, previous studies have shown that RMI violations occur only when the two target attributes belong to the same object (or nearby objects, less than some $4^{\circ}$ of visual angle apart; Feintuch \& Cohen, 2002; Krummenacher et al., 2002a), indicating that only signals that emerge from the same (or nearby) spatial location(s) co-activate. Capitalizing on the spatial restrictions on co-activation, a potentially more sensitive index of co-activation (and therefore a stricter test for the absence of co-activation) is the difference in RTs between a condition with one redundant target (redundant/same location) and a condition in which 
both target attributes are also present, but in different objects (redundant/different locations). If there is a simple race between the two attributes (i.e., if the two signals are not integrated in any way), it should not matter whether they are present at the same or at different locations. Shorter RTs in the same-location condition (relative to the different-locations condition) would indicate that it matters whether the two signals can be integrated on a spatially organized saliency map (Feintuch \& Cohen, 2002; Krummenacher et al., 2002a); accordingly, such a pattern of RTs is incompatible with a simple race.

A further common finding in visual search is that performance improves when the target-defining dimension repeats across consecutive trials (e.g., orientation $\rightarrow$ orientation), relative to dimension changes (e.g., color $\rightarrow$ orientation). Repeating the precise target features (e.g., left-tilted $\rightarrow$ lefttitled) yields only marginally faster responses than just repeating dimensions (right-tilted $\rightarrow$ left-titled), indicating that this effect is largely dimension-specific, rather than feature-specific, in nature (Found \& Müller, 1996; Müller, Heller, \& Ziegler, 1995; Müller, Reimann, \& Krummenacher, 2003). These dimension-repetition effects probably reflect an amplified influence of the previously relevant dimension on saliency signals at the master-map level and the stronger signal, in turn, triggers faster target selection (dimension weighting; Rangelov, Müller, \& Zehetleitner, 2011a,b, 2012; Rangelov, Töllner, Müller, \& Zehetleitner, 2013; Töllner, Gramann, Müller, Kiss, \& Eimer, 2008).

Finally, an additional intertrial-sequence effect emerges in visual-search tasks with redundant targets: responses on single-target trials are slower following redundant-target trials (e.g., color-and-orientation $\rightarrow$ color), relative to dimensionrepetition single-target trials (e.g., color $\rightarrow$ color; redundancy costs; Krummenacher et al., 2001, 2002a,b). While dimension-repetition effects demonstrate that spatial attention can be biased in favor of processing one or the other stimulus dimension (dimension weighting), the existence of redundancy costs demonstrates that a bias towards one dimension comes at a cost for other dimensions. This adds to the evidence from RMI violations that different stimulus dimensions are not processed independently.

\section{Experiment 1}

To test whether mechanisms generating feature-singleton popout generalize to change pop-out, we employed a version of the change-detection task tailored for examining (i) redundancy gains, (ii) co-activation, (iii) dimension-repetition effects, and (iv) redundancy costs. To test these effects, the change could be either redundant (color and orientation) or single (color only or orientation only), with redundant changes appearing either at the same location or at different locations.
If feature-singleton pop-out and change pop-out are generated by similar mechanisms, all four effects should emerge in the change-detection task.

\section{Methods}

Participants Thirty-two healthy human adults took part in the experiment for either course credits or monetary remuneration $(8 € / h)$. Visual-search studies that robustly observe RMI violations and intertrial-sequence effects typically employ between eight and 16 participants (e.g., Krummenacher et al., 2001, 2002a,b). To detect even potentially weaker effects in change detection (for which no prior experience is available), we doubled the higher of these numbers. All participants had normal or corrected-to-normal visual acuity and normal color vision (as confirmed by Ishihara color plates). All participants had extensive experience with psychophysical experiments and all were naïve as to the purpose of the study. The experiment was approved by the responsible ethics committee of the LMU Munich, and all participants provided informed consent prior to the experiment. QUEST did not converge for four participants (see below) and two participants did not finish the experiment. These participants were removed from subsequent data analyses. The final sample thus consisted of 26 participants (mean age: 25 years; 16 female). This is still larger than Saiki's (2016) sample $(N=23)$, who recently observed RMI violations in a different visual working-memory task.

Stimuli and design Stimulus presentation (TFT screen, 1,920 $\times 1,080$ pixels, $60 \mathrm{~Hz}$, viewing distance $=60 \mathrm{~cm}$ ) and response collection ( $\mathrm{F}$ and $\mathrm{J}$ key on a computer keyboard) was controlled by a Matlab (The Mathworks, Natick, MA, USA) program, using functions from the Psychophysics Toolbox (Brainard, 1997). Stimuli were colored and tilted arrowheads, $1.5^{\circ}$ of visual angle in width, randomly positioned on an imaginary circle $\left(6^{\circ}\right.$ radius, $4^{\circ}$ minimal distance between stimuli) around a central fixation cross $\left(0.5^{\circ}\right)$ on a dark grey background. Colors were randomly drawn from a circle in a luminance plane of the CIE Lab space ( $L=63$, center: $a=9, b=$ 27). Lab values were chosen to allow for large radii (up to 40) with colors displayable in SRGB space. Radii were 20 in the first phase and scaled in the second phase (see below). On each trial, one color and one orientation were selected randomly from the 360 possible values. The remaining colors and orientations (if applicable) were then selected randomly from a pool of six values $\left(60^{\circ}\right.$ steps, starting at the original random value), so that any two arrowheads (including the change) differed minimally by $60^{\circ}$ in color and orientation.

Each trial (Fig. 1) consisted of a memory array, presented for $200 \mathrm{~ms}$, containing three to-be-remembered arrowheads. After a blank retention period of $1,000 \mathrm{~ms}$, the test array containing three arrowheads was presented until response or maximally $1,500 \mathrm{~ms}$. This was followed by written feedback for 


\section{Experiment 1}

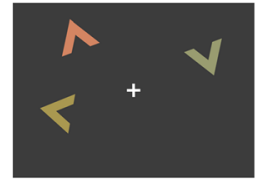

Memory Array

(200 ms)

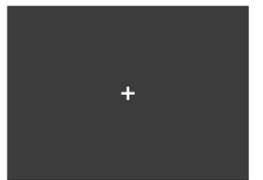

Retention Interval

$(1,000 \mathrm{~ms})$

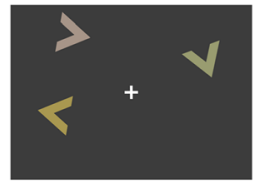

Test Array

(max. 1,500 ms)

\section{Experiment 2}

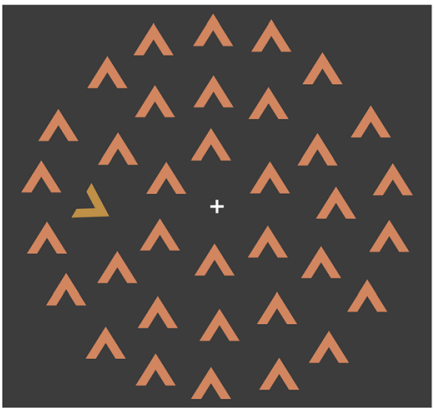

Search Display

(max. 1,500 ms)
Fig. 1 Illustration of the display sequence in Experiment 1 and the search display in Experiment 2. In Experiment 1, participants reported whether any of the elements in the test array differed from the memorized elements. The example depicts a redundant change at the same location: both the color and the orientation of the upper left element changed in the

late or incorrect responses (1,500 ms; "Faster!" or "Wrong!" in German) and, finally, a blank intertrial interval (800-2,000 $\mathrm{ms})$. The fixation cross was visible throughout the trial, except for the feedback. Participants reported, as fast and accurately as possible, whether any element differed between memory and test arrays via key presses (left/right index finger, response-assignment counterbalanced across participants), and the timing and accuracy of these presses was recorded. After each block, participants received feedback on their mean accuracy.

The experiment consisted of three phases. To measure the difficulty of detecting orientation changes, individual arrowheads differed in orientation and only one orientation could change (by $\pm 120^{\circ}, 2 / 3$ of trials) in the first phase (two blocks of 51 trials). All arrowheads in a display had the same color (varying randomly across trials). In the second phase (two blocks of 51 trials), the QUEST scaling algorithm (KingSmith, Grigsby, Vingrys, Benes, \& Supowit, 1994; Watson $\&$ Pelli, 1983) was used $(\beta=3.5, \delta=0.01, \gamma=0.5)$ to match the difficulty of detecting color changes to that of detecting orientation changes. In this phase, the three arrowheads differed in color (while having the same random orientation), and only one color could change (by $\pm 120^{\circ} ; 2 / 3$ of trials). The discriminability of color changes was adjusted by changing the radius of the color circle (guess $=20$, range $=0-40$ ). The aim of the first two phases was to equate the difficulty of detecting the two types of change. The first phase measured the difficulty of detecting $120^{\circ}$ orientation changes and the second phase adapted the difficulty of detecting $120^{\circ}$ color changes accordingly. ${ }^{1}$ Thus, all participants performed the task with orientation changes first and the task with color

\footnotetext{
${ }^{1}$ Adapting detection difficulty, of course, requires that orientation changes are sufficiently difficult to detect, so that detection performance is not at ceiling (i.e., that the QUEST threshold is clearly below $100 \%$ correct). We ascertained below-ceiling performance for orientation changes in pilot tests.
}

test relative to the memory array. In Experiment 2, participants reported whether a singleton target was present. Here too, the example depicts a redundant target at the same location: both color and orientation of the target object differ from the surrounding distractor objects

changes second, without counterbalancing. This resulted in color-circle radii from 2.96 to 35.68 (mean $=17.69)$. Data from these two phases were not further analyzed.

In the third, main phase (12 blocks of 66 trials), each arrowhead was uniquely colored and oriented (see Fig. 1). The memory and probe arrays were identical (no change) on $1 / 3$ of the trials. On half of the remaining trials, one, randomly selected arrowhead changed in either color or orientation (single change). On the other half, there was both a color and an orientation change (redundant changes). On half of the redundant-change trials, two arrowheads changed, one in color and the other in orientation (redundant/different locations, mean distance: $9.17^{\circ}$ ). On the remaining half of redundantchange trials, one arrowhead changed in both color and orientation (redundant/same location). Conditions were balanced per block of trials and otherwise completely randomized. Thus, each block consisted of 11 trials per change type and 22 no-change trials presented in a random order.

\section{Results}

Here, we report analyses of the data from the main phase. For analyses on RTs, only correct-response trials were considered (78\% of trials on average across participants and experimental conditions). To detect trials with outlier RTs, we employed Tukey's cut-off rule (e.g., Hoaglin, Iglewicz, \& Tukey, 1986), which uses the lower $\left(F_{L}\right)$ and upper $\left(F_{U}\right)$ RT quartiles: correct trials with RTs faster than $\mathrm{F}_{\mathrm{L}}-1.5\left(\mathrm{~F}_{\mathrm{U}}-\mathrm{F}_{\mathrm{L}}\right)$ or slower than $\mathrm{F}_{\mathrm{U}}+1.5\left(\mathrm{~F}_{\mathrm{U}}-\mathrm{F}_{\mathrm{L}}\right)$ were considered outliers and removed from analyses ( $3 \%$ of correct-response trials on average across participants and experimental conditions). As Table 1 shows, participants responded slowest on no-change trials, followed by single-change trials, and fastest on redundant-change trials. Error rates were lowest for redundant changes, while the other experimental conditions differed little in error rates. 
Table 1 Results for each experimental condition (Change Type) and split according to intertrial sequence (Trial $n-1 \rightarrow$ Trial $n$ ). Numbers in parentheses indicate $95 \%$-within-subjects confidence intervals for the main effect of Change Type or Intertrial Sequence, respectively, according to Loftus and Masson (1994), or the interquartile range (for the number of trials)

\begin{tabular}{llll}
\hline & Median RTs $(\mathrm{ms})$ & Error rates $(\%)$ & \\
\hline Change type & & & \\
No change & $669(662-676)$ & $28(26-30)$ & 264 \\
Single/Color & $606(599-613)$ & $29(27-31)$ & 132 \\
Single/Orientation & $631(624-638)$ & $12(10-14)$ & 132 \\
Redundant/Same location & $575(568-582)$ & $10(8-12)$ & 132 \\
Redundant/Different locations & $573(566-580)$ & & 132 \\
Intertrial sequence & & $26(25-27)$ & $24(23-25)$ \\
Single change $\rightarrow$ Single change & & $28(27-29)$ \\
Dimension repetition & $613(605-621)$ & $10(9-11)$ & $40(34-43)$ \\
Dimension change & $620(612-628)$ & $11(10-12)$ & $86(39-47)$ \\
Redundant $\rightarrow$ Single & $606(598-614)$ & $83-93)$ \\
Single $\rightarrow$ Redundant & $573(565-581)$ & $86(79-95)$ \\
Redundant $\rightarrow$ Redundant & $574(566-582)$ &
\end{tabular}

$R T$ response time

These observations were supported by Holm-adjusted pairwise $t$ tests, which revealed significantly slower RTs for the no-change condition relative to all change conditions (all $t(25) \mathrm{s} \geq 3.30$, all $p \mathrm{~s} \leq .009$, all Cohen's $d_{\mathrm{z}} \mathrm{s} \geq 0.66$ ). Error rates, by contrast, were similar for no-change and single-change conditions (both $t(25) \mathrm{s} \leq 1.41$, both $p \mathrm{~s}>.250$, all $d_{\mathrm{z}} \mathrm{s} \leq$ 0.28 ). While single color changes were detected significantly faster than single orientation changes $(t(25)=3.07, p=.010$, $\left.d_{\mathrm{z}}=0.61\right)$, they were detected with similar accuracy $(t(25)=$ $1.48, p>.250, d_{\mathrm{z}}=0.30$ ), indicating successful stimulus scaling. The faster (color) single change was significantly slower than redundant changes at either the same location $(t(25)=$ $\left.5.13, p<.001, d_{\mathrm{z}}=1.03\right)$ or different locations $(t(25)=4.92, p$ $\left.<.001, d_{\mathrm{z}}=0.98\right)$, indicating significant redundancy gains. Similarly, error rates for single changes were significantly higher than for redundant changes at either the same or different locations (both $t(25) \mathrm{s} \geq 6.24$, both $p<.001$, both $d_{\mathrm{z}} \geq$ 1.25). Interestingly, there were no significant differences between redundant changes at the same and at different locations, in either RT $\left(t(25)=.32, p>.250, d_{\mathrm{z}}=0.06\right)$ or error rate $\left(t(25)=2.30, p=.120, d_{z}=0.46\right)$. Taken together, analyses of median RTs and error rates revealed substantial redundancy gains of similar magnitude for changes at the same and at different locations.

To understand better what these redundancy gains reflect, we tested for violations of the race-model inequality (RMI). Violations of the RMI are regarded as evidence for co-active processing, in which case rejecting the simpler race model is warranted. To test for RMI violations, we estimated RTs at ten percentiles of the cumulative distribution function (CDF) for each condition as well as the sum of the two single-change CDFs (5th-95th percentile in steps of 10) according to the method of Ulrich, Miller, and Schröter (2007). The sum of the two single-change CDFs constitutes the theoretical upper bound that is still compatible with race models. If, at any percentile, RTs in a redundant-signal condition are faster than the upper bound (i.e., the RMI is violated), independent race processing can be reliably rejected. As Fig. 2 shows, for both redundant-change conditions, none of the percentile RTs was faster than the race-model bound, that is, there were no violations of the RMI. In fact, two-tailed paired $t$ tests (corrected for multiple-comparisons by the method of Hochberg, 1988) revealed that all but the fastest percentile RTs for redundant changes at both the same and different locations were significantly slower than the race-model bound. Nevertheless, consistent with the redundancy gains observed in median RTs (the 50th percentile), redundant changes at both the same and different locations were detected significantly faster than single color changes for all percentiles.

As mentioned earlier, RMI violations are a conservative criterion for co-activation, permitting reliable rejection of race models. Simple absence of RMI violations, however, does not permit rejecting any of the models. As a more sensitive test of co-activation, we compared RT distributions for redundant changes at the same and different locations. If the changes are indeed detected at the master-map level (similar to the master map of saliencies), then redundant changes at the same location would co-activate, predicting faster RTs. A simple race, by contrast, predicts no differences between redundant changes at the same location and at different locations. Consistent with race-model predictions, Fig. 2 shows a striking overlap between RT distributions in these two conditions. This observation was confirmed by analyses of Bayes factors (BF) for a mixed-measures linear model (Morey, Rouder, \& 


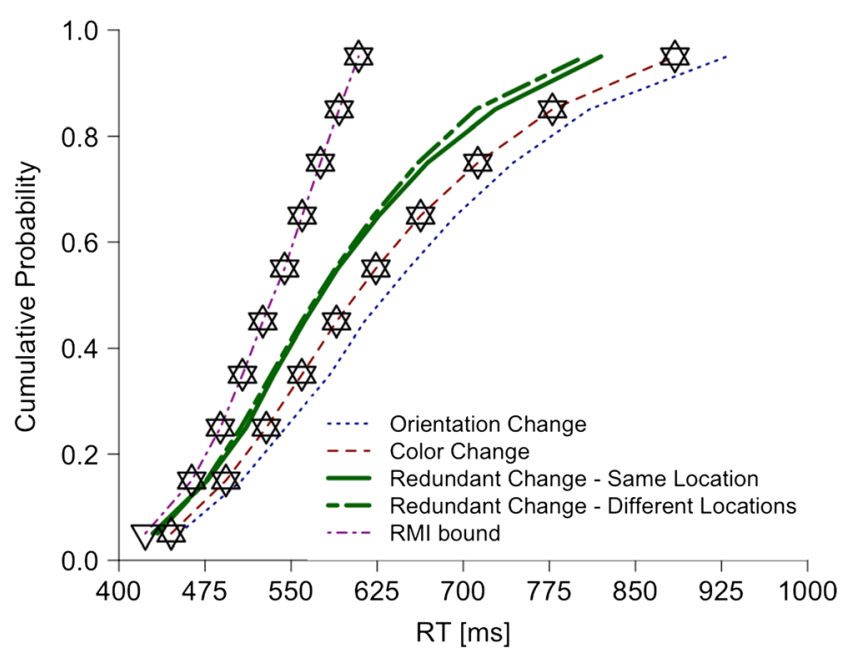

Fig. 2 Response time (RT) distributions for different change types in Experiment 1 (change detection task). Upward-pointing triangles denote significant differences (Hochberg-corrected, two-tailed $p<.05$ ) from redundant changes at the same location, whereas downward-pointing triangles denote significant differences from redundant changes at different locations. Asterisks are a combination of two (upward and downwardpointing) triangles

Jamil, 2015) of RT distributions for redundant targets with change type (same vs. different locations) as a categorical predictor, cumulative probability as a continuous predictor, and participant as a random factor. We examined $2 \ln (\mathrm{BF})$ values, which have the attractive property that their absolute value can be interpreted in the same way irrespectively of whether the data support the null $(2 \ln (\mathrm{BF}) \leq-2)$ or the alternative $(2 \ln (B F) \geq 2)$ hypothesis (Kass \& Raftery, 1995). The $2 \ln$ (BFs) values supported the null hypothesis that the RT distributions for redundant changes at the same versus different locations were equivalent in both the fastest RTs $(2 \ln (\mathrm{BF})$ $=-4.38$ for the main effect of change type) and RT variability $(2 \ln (\mathrm{BF})=-4.72$ for the interaction between the change type and cumulative probability).

Analyses of intertrial-sequence effects on RTs (Table 1) revealed none of the sequence effects typically observed in singleton-feature search. Dimension-repetition and dimension-change trials did not differ (i.e., there were no dimension-repetition effects) in median RTs $(2 \ln (\mathrm{BF})=$ -2.73). Redundancy costs were absent, too: single-change trials following a redundant-change trial were, if anything, responded to faster than dimension-repetition trials. Inspection of the error rates revealed no indications of speed-accuracy trade-offs across conditions. Taken together, intertrial-sequence effects (dimension-repetition effects and redundancy costs), which are robust in visual-search tasks, were absent in the change-detection task.

\footnotetext{
${ }^{0}$ In two pilot studies, we used only three or six items in a visual search task and observed no pop-out (i.e., there was a clear effect of set size) for even a very strong orientation contrast (of $120^{\circ}$ ).
}

\section{Discussion}

We transferred a redundant-signals paradigm typically used to examine the workings of saliency maps in visual search to the change-detection task to test whether saliency computations are similar across tasks.

On the one hand, we found clear and robust redundancy gains in both redundant-change conditions (faster RTs for redundant compared with single changes). Such effects are typically explained by a parallel processing (i.e., a race) of two saliency signals: the response is triggered as soon as one signal finishes processing without having to wait for the other signal (Miller, 1982). Thus, the substantial redundancy gains for the change detection task we observed in Experiment 1 support the notion that changes are detected in parallel akin to pop-out search. This nicely complements the evidence for change popout provided by Hyun et al. (2009). Notably, both phenomena (race and pop-out) are typically explained by a parallel processing on saliency maps.

On the other hand, however, no sign of co-activation or intertrial-sequence effects - that is, effects that are diagnostic of the hierarchical architecture of saliency computation in visual search - emerged in our change-detection task. These striking deviations from standard findings reported in the visual-search literature (e.g., Feintuch \& Cohen, 2002; Krummenacher et al., 2001, 2002a,b) indicate fundamental differences in the way saliency maps are employed in the two tasks.

\section{Experiment 2}

Rather than assuming fundamental differences between processes underlying change detection and visual search, one could maintain that the specifics of our stimuli and experimental design are responsible for the deviating data pattern. In other words, it is conceivable that using a comparable stimulus set and experimental design, the co-activation and intertrialsequence effects would likewise be absent in a visual-search task. To rule out this possibility and to provide a more direct comparison between the two tasks, in Experiment 2 we administered a visual-search task similar to the change detection task used in Experiment 1.

Given that change detection and visual search differ in many respects, a direct translation of a change-detection design to a visual-search design is, of course, not possible. For instance, error rates in change-detection are necessarily higher, because information might be lost from memory before the test array comes up and search can commence, whereas all necessary information is physically available in a standard visual-search task. Perhaps most importantly, performance in the two tasks relies on different types of contrast signals. Change detection, on the one hand, depends on the 
(change) contrast between a target (the changed object) and an object at the same position stored in VWM. In pop-out search, on the other hand, target detection depends on the (feature) contrast between the target (a feature singleton) and concurrently presented surrounding distractor objects (Duncan \& Humphreys, 1989; Liesefeld et al., 2016; Nothdurft, 2000; Rangelov, Müller, \& Zehetleitner, 2017).

These differences have two main implications for the design of Experiment 2. First, previous research (e.g., Rangelov et al., 2017) as well as unpublished evidence ${ }^{2}$ suggest that, in order to achieve reliable pop-out in visual search, more objects must be in the display as compared to change detection. Additionally, employing only three items is problematic, because in the redundant/different-locations condition all three objects differ from each other, so that all objects are singletons (i.e., the two targets are not special).

Second, a given change contrast might not directly translate into a respective feature contrast. For instance, a change from blue to green might not directly compare to the contrast between a blue target and surrounding green distractors. This loss in translation might have unfavorable consequences for the present visual-search experiment: if one contrast (e.g., color) is much stronger than the other (e.g., orientation), participants likely focus on the more efficiently processed dimension (e.g., color) and ignore the other dimension (e.g., orientation) in a first stage (and switch over only once they have established the absence of a color target). Consequently, the same signal (color) would always determine response times, that is, no redundancy gains would emerge. In fact, this is what we found when we directly translated the contrasts in a first attempt to reproduce the typical visual-search findings in a dense display. Without redundancy gains, one might question the comparability to typical visual-search tasks and to the change-detection task in Experiment 1. Accordingly, to assure that there is a race in which both signals have a chance to win, we determined, in a preliminary scaling study, a color contrast that is comparable to the orientation contrast.

Thus, for Experiment 2, we decided to use dense displays and color targets that were detected comparably fast relative to orientation targets (as determined in an independent scaling experiment, which tested a range of color contrasts).

\section{Methods}

Participants Twenty healthy human adults took part in the experiment for either course credits or monetary remuneration $(8 € / h)$. Three participants missed the single-color target on more than $50 \%$ of trials and were, thus, excluded from further analysis. The final sample consisted of 17 participants (mean

\footnotetext{
${ }^{2}$ In two pilot studies, we used only three or six items in a visual search task and observed no pop-out (i.e., there was a clear effect of set size) for even a very strong orientation contrast (of $120^{\circ}$ ).
}

age: 22 years; 12 female). This is at the upper end of sample sizes in typical redundant-signals studies (e.g., Krummenacher et al., 2001, 2002a,b).

Stimuli and design Stimulus presentation (TFT screen, 1,920 $\times 1,080$ pixels, $60 \mathrm{~Hz}$, viewing distance $=60 \mathrm{~cm}$ ) and response collection ( $\mathrm{F}$ and $\mathrm{J}$ keys on a computer keyboard) was controlled by a Matlab (The Mathworks) program, using functions from the Psychophysics Toolbox (Brainard, 1997). Search displays in the main part of the experiment consisted of 36 colored arrowheads, $1.5^{\circ}$ of visual angle in width, arranged with a positional jitter of $\pm 0.2^{\circ}$ on 3 concentric imaginary circles with radii of $2^{\circ}, 4^{\circ}$, and $6^{\circ}(6,12$, and 18 arrowheads), respectively, around a central fixation cross $\left(0.5^{\circ}\right)$ on a dark grey background, as illustrated in Fig. 1. Two colors (one for targets and one for non-targets) were drawn from a circle in the same luminance plane of the CIE Lab space as in Experiment $1(\mathrm{~L}=63$, center: $\mathrm{a}=9, \mathrm{~b}=27)$ with a radius of 17.69 (the average across participants from Experiment 1). Non-target arrowheads were orange $(a=25.61, b=33.05)$ and pointed upwards. The target color was a different shade of orange ( $\mathrm{a}=21.50, \mathrm{~b}=39.51$; i.e., $5^{\circ}$ clockwise from the non-target color on the color circle), and the target orientation was a $120^{\circ}$-tilt from upright. As mentioned above, the color contrast was chosen based on data from an independent scaling experiment, so as to equate the detection speeds for color and orientation targets and, thereby, to assure that both features are processed.

Each search display was presented until response or maximally $1,500 \mathrm{~ms}$. The fixation cross was visible throughout the trial and turned blue for late and, respectively, red for incorrect responses $(1,500 \mathrm{~ms})$. Trials were separated by a blank interval (800-2,000 ms). Participants reported, as fast and as accurately as possible, whether any target object was present (vs. absent) via key press (left/right index finger, responseassignment counterbalanced across participants), and the timing and accuracy of these presses was recorded. After each block, participants received feedback on their mean accuracy. Displays contained either a single color target, a single orientation target, both a color and an orientation target (with at minimum one non-target object in between the two targets), or a target redundantly defined by color and orientation. Within each block, each target condition occurred on $1 / 6$ of all trials; on $1 / 3$ of trials no target was present. All conditions were randomly intermixed. Participants performed a (nonanalyzed) practice block of 24 trials, followed by 21 blocks of 96 trials each.

After the main part, participants performed an additional task with only single-color and single-orientation targets and a set-size manipulation. Now displays contained 19 or 37 objects (an additional object was present in the center of the array, replacing the fixation cross). Participants were told that the outer ring (containing 12 or 18 objects) would never 
contain a target, so that it is reasonable to assume that they ignored it (did not suspect a target on this ring), resulting in effective set sizes of seven or 19 objects, respectively (see Liesefeld et al., 2016). The purpose of the outer ring was to assure that all targets (including those on the second-toouter ring) were surrounded by homogenous non-targets and would therefore exhibit high feature contrast (Liesefeld et al., 2016; Nothdurft, 1993, 2000). The whole array was shifted at random on each trial so that target eccentricity was, on average, kept constant between the two set sizes (see Liesefeld et al., 2016, Experiments 2 and 3). All other stimulation details were identical to the main part of Experiment 2. Experimental conditions were intermixed and balanced within each block according to a full factorial target presence (present vs. absent) $\times$ target type (color vs. orientation) $\times$ set size (seven vs. 19) design. Participants performed three blocks of 96 trials each.

\section{Results}

Similar to Experiment 1, for analyses on RTs, only correctresponse trials were considered. Outlier RTs were rejected using Tukey's outlier-detection criterion as detailed above (5\% of trials on average across participants and experimental conditions). Effects of experimental conditions on median RTs and error rates were again tested for significance using Holmadjusted pairwise $t$ tests. We first analyzed the set-size effect on correct-response RTs to test whether the color and orientation targets indeed produced pop-out. Set-size effects were small (set-size effects smaller than $5 \mathrm{~ms} /$ item are usually considered evidence for parallel search/pop out; Liesefeld et al., 2016; Treisman \& Souther, 1985; Wolfe, 1998) and nonsignificant for both color, $0.0 \mathrm{~ms} /$ item, $t(16)=0.01, p=$ $.995, d_{\mathrm{z}}<0.01$, and orientation, $1.5 \mathrm{~ms} /$ item, $t(16)=1.90, p$ $=.075, d_{\mathrm{z}}=0.46$. There was also no effect of set size on error rates (both $t(16) \mathrm{s} \leq 1.78$, all $p \mathrm{~s}>.096$, all Cohen's $d_{\mathrm{z}} \mathrm{s}<0.43$ ).

In the main part of the experiment, participants again responded slowest on target-absent trials, all $t(16) \mathrm{s} \geq 7.76$, all $p \mathrm{~s} \leq .001$, all Cohen's $d_{\mathrm{z}} \mathrm{s} \geq 1.88$ (see Table 2). Importantly, responses were faster on redundant-target as compared to single-target trials, all $t(16) \mathrm{s} \geq 3.86$, all $p \mathrm{~s} \leq$ .003 , all Cohen's $d_{\mathrm{z}} \mathrm{s} \geq 0.94$. That is, we again observed clear redundancy gains. In contrast to Experiment 1, redundant signals at the same location were detected faster than redundant signals at different locations, $t(16)=7.05, p<.001, d_{\mathrm{z}}=1.68$, indicating co-activation. As a consequence of our scaling of contrast values for equating RTs, orientation targets and color targets were detected equally fast, $t(16)=0.57, p=.576, d_{\mathrm{z}}=$ 0.14 . Error rates were lowest for redundant targets, all $t(16) \mathrm{s} \geq$ 5.32, all $p \mathrm{~s} \leq .001$, all Cohen's $d_{\mathrm{z}} \mathrm{s} \geq 1.33$, and did not differ between the two redundant-target conditions, $t(16)=0.22, p=$ $.830, d_{\mathrm{z}}=0.05$. Error rates for target-absent trials were lower than those for color targets, $t(16)=4.65, p=.001, d_{\mathrm{z}}=1.16$, but similar to those for orientation targets, $t(16)=1.02, p=$ $646, d_{\mathrm{z}}=0.25$. In contrast to RTs, error rates differed between the two single-target conditions, $t(16)=4.12, p=.002, d_{\mathrm{z}}=$ 1.03 .

To follow up on the redundancy gains in RTs, we again tested for RMI violations. As Fig. 3 shows, for both redundant-target conditions, none of the percentile RTs was shorter than any of the respective percentile RTs from the RMI bound, that is, there were no violations of the RMI. However, several percentile RTs were shorter for the redundant/samelocation as compared to the redundant/different-locations condition, thus indicating co-activation in the absence of RMI violations.

Analyses of intertrial-sequence effects on RTs (Table 2) revealed all expected sequence effects. Responses were faster for dimension-repetition than for dimension-change trials, $t(16)=4.95, p<.001, d_{\mathrm{z}}=1.24$, and redundancy costs (slowing for single targets following a redundant-target relative to dimension-repetition targets) were also manifest, $t(16)$ $=3.48, p=.009, d_{\mathrm{z}}=0.87$. Analyses of intertrial-squence effects on error rates revealed a similar pattern, showing dimension-repetition effects, $t(16)=3.82, p=.006, d_{\mathrm{z}}=$ 0.95 , and redundancy costs, $t(16)=3.16, p=.018, d_{\mathrm{z}}=0.79$.

\section{Discussion}

In Experiment 2, we used a visual-search task with stimuli and a design similar to that of the change-detection task in Experiment 1. In contrast to Experiment 1, we observed most standard findings from the visual-search literature that have been taken as evidence for a master map of saliencies. Taken together, the results of Experiment 2 suggest that the lack of intertrial and co-activation effects in Experiment 1 was not simply due to a specific stimulus set and experimental design. Of note, though, we did not replicate one much stressed finding from the visual-search literature: the RT distribution for redundant/same-location targets did not violate the RMI. However, tests for RMI violations are known to be rather conservative, that is, these tests might miss co-activation when it is actually present (e.g., Ratko-Dehnert, 2013). As argued above, the difference between RTs for redundant/samelocation and redundant/different-locations targets provides a more sensitive test for co-activation in visual search, and this difference turned out to be robust in the present experiment.

\section{General discussion}

We examined whether searching for a change between two successively presented scenes (change detection) is governed by the same principles as searching for an element in a currently viewed scene (visual search). On the one hand, we observed robust redundancy gains, which complement the 
Table 2 Results for each target type and split according to intertrial sequence (Trial $n-1 \rightarrow$ Trial n). Numbers in parentheses indicate 95\%-withinsubjects confidence intervals for the main effect of Change Type or Intertrial Sequence, respectively, according to Loftus and Masson (1994), or the interquartile range (for the number of trials)

\begin{tabular}{llll}
\hline & Median RTs $(\mathrm{ms})]$ & Error rates $(\%)$ & Trials \\
\hline Change type & & & \\
No change & $688(679-697)$ & $4.11(3.50-4.72)$ & $7.48(6.87-8.09)$ \\
Single/Color & $585(576-594)$ & $3.53(2.92-4.14)$ & 336 \\
Single/Orientation & $590(581-599)$ & $1.10(.49-1.71)$ & 336 \\
Redundant/Same location & $532(523-541)$ & $1.06(.45-1.67)$ & 336 \\
Redundant/Different locations & $561(552-570)$ & & 336 \\
Intertrial sequence & & & $96(89-102)$ \\
Single change $\rightarrow$ Single change & & $3.87(3.24-4.50)$ & $100(97-106)$ \\
Dimension repetition & $564(558-570)$ & $7.02(6.39-7.65)$ & $202(192-2012)$ \\
Dimension change & $593(587-599)$ & $5.46(4.83-6.09)$ & $216(200-226)$ \\
Redundant $\rightarrow$ Single & $578(572-584)$ & $1.20(.57-1.83)$ & $204(200-208)$ \\
Single $\rightarrow$ Redundant & $537(531-543)$ & $.77(.14-1.40)$ & \\
Redundant $\rightarrow$ Redundant & $533(527-539)$ & & \\
\hline$R T$. & & & \\
\hline
\end{tabular}

$R T$ response time

findings of Hyun et al. (2009) and further support the notion that change detection relies on some sort of saliency map (see Discussion of Experiment 1). Critically, however, dimensionrepetition effects, indices of co-activation, and intertrial redundancy costs (i.e., effects reliably found in visual search) were absent in the change-detection task. This indicates that key mechanisms at play in visual search (dimension weighting, co-activation) play no role in change detection. Instead, our findings suggest that similar, yet distinct, mechanisms mediate pop-out in visual-search and change-detection tasks, respectively (see Fig. 4).

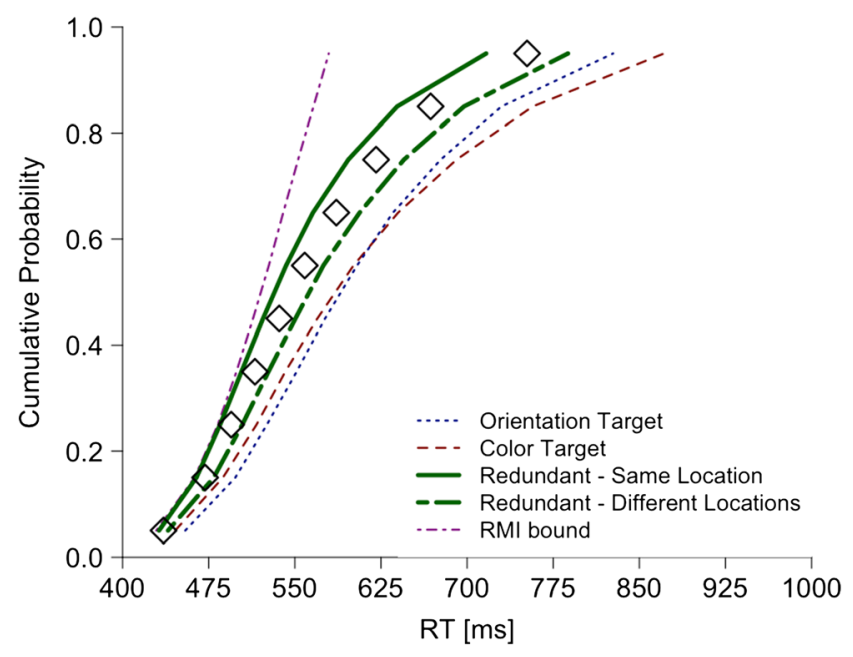

Fig. 3 Response time (RT) distributions for different target types in Experiment 2 (visual search task). Diamonds denote significant differences (Hochberg-corrected, two-tailed $p<.05$ ) between redundant changes at the same location and at different locations
As an interesting aside, we observed no difference in error rates between the two redundant-change conditions; this finding could be taken as tentative evidence that binding plays no major role during VWM maintenance. If all features of an object are maintained (and forgotten) together, the probability that any of the two changed objects in the redundant/differentlocations condition is remembered should be higher than the probability that the single object in the redundant/samelocation condition is remembered (e.g., Bays, Wu, \& Husain, 2011; Rangelov \& Zeki, 2014). However, changedetection performance does not only depend on whether the changed object is maintained, but also on the magnitude of the change and, thus, the discriminability of changes versus nonchanges (Awh, Barton, \& Vogel, 2007). Accordingly, the larger change in one object in the redundant/same-location condition (two features instead of only one) might have offset the advantage of two changing objects in the redundant/differentlocations condition. The present findings thus contribute another interesting indication to the debate on the status of objects during VWM maintenance, while not being decisive on their own.

That a change pops out, as demonstrated by Hyun et al. (2009), is actually more fascinating than meets the eye: How can attention be guided by something that is not present but merely defined as the difference between two subsequently presented displays (a change)? Attention could not be guided by any feature of the changed stimulus: its features were not known in advance and the changed item was special only in that it was not present in the memory display. In fact, if participants had used their VWM content to guide search, they would have attended anywhere else but the change location, 


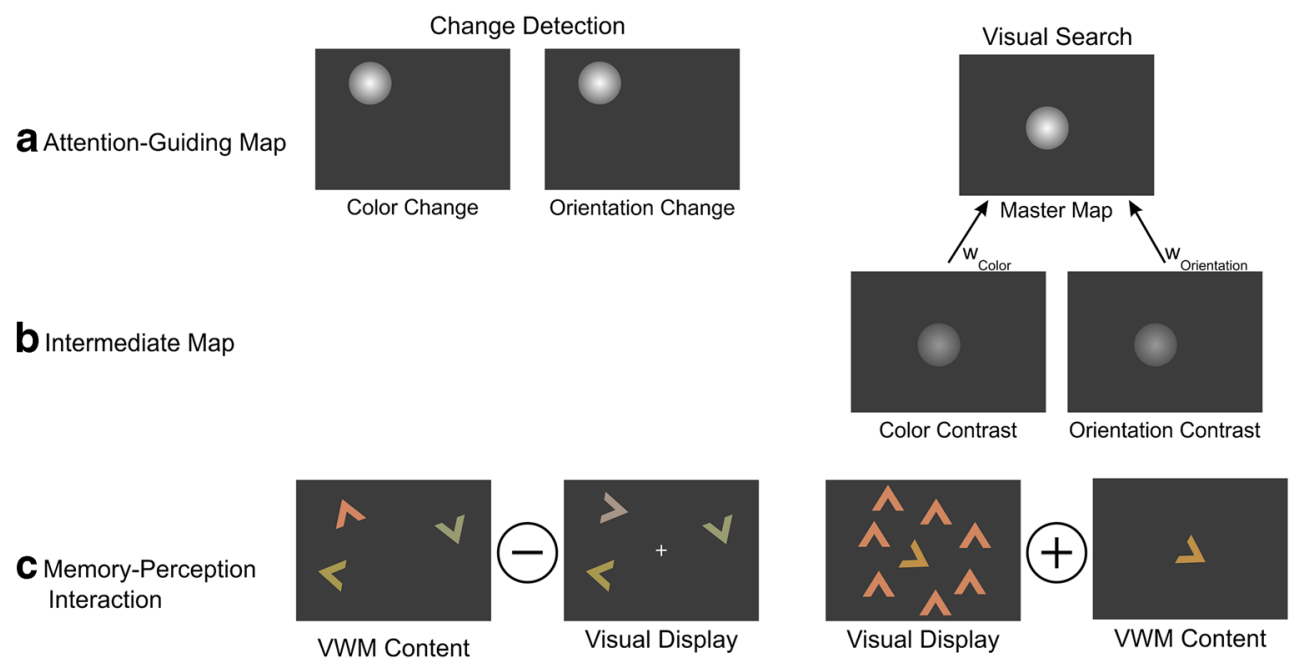

Fig. 4 Mechanisms that potentially mediate pop-out in change-detection and visual-search tasks, respectively. Signals from (a) attention-guiding maps direct attention to the location of the highest saliency. The absence of intertrial effects and race-model inequality (RMI) violations in change detection suggest that there is no (weighted) integration of changecontrast signals at some superordinate, "master-map" level. For visual search, the intertrial effects and RMI violations support the notion that signals from different perceptual dimensions are (b) first processed separately and then integrated as a weighted sum at the level of the supra-dimensional saliency map. Change contrasts depend on (c) feature differences between memorized and currently present items at the same location, whereas feature contrasts depend on local differences between (nearby) items within a given display. The depicted visual-search display is a cutout from the original display of Fig. 1. Feature-contrast signals are potentially enhanced for items that match the attentional template, as compared to non-matching items because the remaining (non-changed) elements matched their VWM content.

When observers use an attentional template to search for a specific object, they are essentially performing an anysameness task in which they compare elements in the search display with the template. Change detection, by contrast, does not require finding matches but finding any differences between two displays. Crucially, any-sameness and anydifference tasks are known to produce data patterns differing in many respects and are likely to involve different cognitive mechanisms (for a review, see Farell, 1985). Hyun et al. (2009), for example, found that any-sameness VWM tasks (tasks in which participants decide whether at least one object remained the same between memory and test) yield much larger search slopes (increases in RT with the number of elements) than the typical any-difference tasks (tasks in which participants decide whether at least one object changed between memory and test). This finding suggests that the target in any-sameness VWM tasks may not pop out. Furthermore, Saiki (2016) recently employed an any-sameness VWM task with redundant targets and found violations of the RMI. Together, ours and Saiki's findings thus further highlight the strong differences between cognitive mechanisms involved in any-sameness and any-difference tasks (for a related discussion on detection vs. discrimination tasks, see Lupiáñez, 2010, and Lupiáñez, Martín-Arevalo, \& Chica, 2013).

Co-activation observed in visual search and the anysameness VWM task suggests that these tasks rely on a feature-blind master-saliency map that represents feature- contrast signals combined across separate dimensions. Change detection, by contrast, might rely on feature-specific maps. One possible account for the present, change-detection results may be that feature maps representing the respective feature in the memory and test displays are compared by subtraction (Fig. 4). Accordingly, each of the resulting difference maps would contain information only about changes in a single feature. The strong mean-RT redundancy gains observed in the present task indicate that signals from several difference maps compete for focal attention; and the absence of intertrial effects and of indices of co-activation indicates that the difference signals are kept separate, rather than being integrated (in a weighted fashion) at some superordinate level. The proposed account is consistent with dominant models of early vision (Wolfe, 2007) and can explain the distinction between anysameness and any-difference tasks in a straightforward manner. While alternative accounts are conceivable too, their respective merits would have to be explored in future studies.

Awh et al. (2012; see also, e.g., Müller et al., 2003) recently proposed that search is always guided by the same master (or priority) map which integrates stimulus-salience, current-goal, and selection-history information. The absence of coactivation observed in the present change-detection data indicates that change maps do not feed into any common priority map, but are instead evaluated independently. The change maps would thus constitute attention-guiding representations that complement the priority map in situations in which the latter does not provide the information required for the task at hand. 
Author Note This work was supported by RA 2191/1-1 grant of the German Research Foundation (DFG), awarded to DR, by MU773/14-1 grant of the DFG awarded to HJM, and by a Junior Research grant from LMU Munich's Institutional Strategy LMUexcellent within the framework of the German Excellence Initiative, awarded to HRL.

\section{References}

Awh, E., Barton, B., \& Vogel, E. K. (2007). Visual working memory represents a fixed number of items regardless of complexity. Psychological Science, 18, 622-628. doi:10.1111/j.1467-9280. 2007.01949.x

Awh, E., Belopolsky, A. V., \& Theeuwes, J. (2012). Top-down versus bottom-up attentional control: A failed theoretical dichotomy. Trends in Cognitive Sciences, 16, 437-443. doi:10.1016/j.tics. 2012.06.010

Bays, P. M., Wu, E. Y., \& Husain, M. (2011). Storage and binding of object features in visual working memory. Neuropsychologia, 49, 1622-1631. doi:10.1016/j.neuropsychologia.2010.12.023

Brainard, D. H. (1997). The Psychophysics Toolbox. Spatial Vision, 10, 433-436. doi:10.1163/156856897X00357

Desimone, R., \& Duncan, J. (1995). Neural mechanisms of selective visual attention. Annual Review of Neuroscience, 18, 193-222. doi:10.1146/annurev.ne.18.030195.001205

Downing, P. E. (2000). Interactions between visual working memory and selective attention. Psychological Science, 11, 467-473. doi:10. 1111/1467-9280.00290

Duncan, J., \& Humphreys, G. W. (1989). Visual search and stimulus similarity. Psychological Review, 96, 433-458. doi:10.1037/0033295X.96.3.433

Farell, B. (1985). 'Same'-'different' judgments: A review of current controversies in perceptual comparisons. Psychological Bulletin, 98, 419-456. doi:10.1037/0033-2909.98.3.419

Feintuch, U., \& Cohen, A. (2002). Visual attention and coactivation of response decisions for features from different dimensions. Psychological Science, 13, 361-369. doi:10.1111/j.0956-7976. 2002.00465.x

Found, A., \& Müller, H. J. (1996). Searching for unknown feature targets on more than one dimension: Investigating a 'dimension-weighting' account. Perception \& Psychophysics, 58, 88-101. doi:10.3758/ BF03205479

Hoaglin, D. C., Iglewicz, B., \& Tukey, J. W. (1986). Performance of some resistant rules for outlier labeling. Journal of the American Statistical Association, 81, 991-999. doi:10.1080/01621459.1986. 10478363

Hochberg, Y. (1988). A sharper Bonferroni procedure for multiple tests of significance. Biometrika, 75, 800-802. doi:10.1093/biomet/75.4. 800

Humphreys, G. W., \& Müller, H. J. (1993). SEarch via Recursive Rejection (SERR): A connectionist model of visual search. Cognitive Psychology, 25, 43-110. doi:10.1006/cogp.1993.1002

Hyun, J., Woodman, G. F., Vogel, E. K., Hollingworth, A., \& Luck, S. J. (2009). The comparison of visual working memory representations with perceptual inputs. Journal of Experimental Psychology: Human Perception and Performance, 35, 1140-1160. doi:10. 1037/a0015019

Kass, R. E., \& Raftery, A. E. (1995). Bayes Factors. Journal of the American Statistical Association, 90, 773-795. doi:10.1080/ 01621459.1995.10476572

King-Smith, P. E., Grigsby, S. S., Vingrys, A. J., Benes, S. C., \& Supowit, A. (1994). Efficient and unbiased modifications of the QUEST threshold method: Theory, simulations, experimental evaluation and practical implementation. Vision Research, 34, 885-912. doi: 10.1016/0042-6989(94)90039-6

Krummenacher, J., \& Müller, H. J. (2014). Visual search for singleton targets redundantly defined in two feature dimensions: Co-active processing of color-motion targets? Journal of Experimental Psychology: Human Perception and Performance, 40, 1926-1939. doi:10.1037/a0037560

Krummenacher, J., Müller, H. J., \& Heller, D. (2001). Visual search for dimensionally redundant pop-out targets: Evidence for parallelcoactive processing of dimensions. Perception \& Psychophysics, 63, 901-917. doi:10.3758/BF03194446

Krummenacher, J., Müller, H. J., \& Heller, D. (2002a). Visual search for dimensionally redundant pop-out targets: Parallel-coactive processing of dimensions is location specific. Journal of Experimental Psychology: Human Perception and Performance, 28, 1303-1322. doi:10.1037/0096-1523.28.6.1303

Krummenacher, J., Müller, H. J., \& Heller, D. (2002b). Visual search for dimensionally redundant pop-out targets: Redundancy gains in compound tasks. Visual Cognition, 9, 801-837. doi:10.1080/ 13506280143000269

Kuhl, B. A., \& Chun, M. M. (2014). Memory and attention. In A. C. Nobre \& S. Kastner (Eds.), The Oxford Handbook of Attention (pp. 753-776). New York: Oxford University Press.

Liesefeld, H. R., Moran, R., Usher, M., Müller, H. J., \& Zehetleitner, M. (2016). Search efficiency as a function of target saliency: The transition from inefficient to efficient search and beyond. Journal of Experimental Psychology: Human Perception and Performance, 42, 821-836. doi:10.1037/xhp0000156

Loftus, G. R., \& Masson, M. J. (1994). Using confidence intervals in within-subject designs. Psychonomic Bulletin \& Review, 1, 476490. doi:10.3758/BF03210951

Luck, S. J. (2012). Electrophysiological correlates of the focusing of attention within complex visual scenes: N2pc and related ERP components. In S. J. Luck \& E. S. Kappenman (Eds.), The Oxford Handbook of ERP Components (pp. 329-360). New York: Oxford University Press.

Lupiáñez (2010). Inhibition of return. In A. C. Nobre \& J. T. Coull (Eds.), Attention and Time (pp. 17-34). Oxford Scholarship Online. doi:10. 1093/acprof:oso/9780199563456.003.0002

Lupiáñez, J., Martín-Arévalo, E., \& Chica, A. B. (2013). Is Inhibition of Return due to attentional disengagement or to a detection cost? The Detection Cost Theory of IOR. Psicológica, 34, 221-252. Retrieved from https://www.uv.es/psicologica/

Miller, J. (1982). Divided attention: Evidence for coactivation with redundant signals. Cognitive Psychology, 14, 247-279. doi:10.1016/ 0010-0285(82)90010-X

Mordkoff, J. T., \& Yantis, S. (1993). Dividing attention between color and shape: Evidence of coactivation. Perception \& Psychophysics, 53, 357-366. doi:10.3758/BF03206778

Morey, R. D., Rouder, J. N., \& Jamil, T. (2015). BayesFactor: Computation of Bayes factors for common designs (Version 0.9.12-2). Retrieved from https://cran.r-project.org/web/packages/ BayesFactor/index.html

Müller, H. J., Heller, D., \& Ziegler, J. (1995). Visual search for singleton feature targets within and across feature dimensions. Perception \& Psychophysics, 57, 1-17. doi:10.3758/BF03211845

Müller, H. J., Reimann, B., \& Krummenacher, J. (2003). Visual search for singleton feature targets across dimensions: Stimulus- and expectancy-driven effects in dimensional weighting. Journal of Experimental Psychology: Human Perception and Performance, 29, 1021-1035. doi:10.1037/0096-1523.29.5.1021

Nothdurft, H. (1993). The role of features in preattentive vision: Comparison of orientation, motion and color cues. Vision Research, 33, 1937-1958. doi:10.1016/0042-6989(93)90020-W 
Nothdurft, H. (2000). Saliency from feature contrast: Variations with texture density. Vision Research, 40, 3181-3200. doi:10.1016/ S0042-6989(00)00168-1

Olivers, C. L., Meijer, F., \& Theeuwes, J. (2006). Feature-based memorydriven attentional capture: Visual working memory content affects visual attention. Journal of Experimental Psychology: Human Perception and Performance, 32, 1243-1265. doi:10.1037/00961523.32.5.1243

Olivers, C. L., Peters, J., Houtkamp, R., \& Roelfsema, P. R. (2011). Different states in visual working memory: When it guides attention and when it does not. Trends in Cognitive Sciences, 15, 327-334. doi:10.1016/j.tics.2011.05.004

Rangelov, D., Müller, H. J., \& Zehetleitner, M. (2011a). Dimensionspecific intertrial priming effects are task-specific: Evidence for multiple weighting systems. Journal of Experimental Psychology. Human Perception and Performance, 37, 100-114. doi:10.1037/ a0020364

Rangelov, D., Müller, H. J., \& Zehetleitner, M. (2011b). Independent dimension-weighting mechanisms for visual selection and stimulus identification. Journal of Experimental Psychology: Human Perception and Performance, 37, 1369-1382. doi:10.1037/ a0024265

Rangelov, D., Müller, H. J., \& Zehetleitner, M. (2012). The multipleweighting-systems hypothesis: Theory and empirical support. Attention, Perception, \& Psychophysics, 74, 540-552. doi:10. 3758/s13414-011-0251-2

Rangelov, D., Müller, H. J., \& Zehetleitner, M. (2017). Failure to pop out: Feature singletons do not capture attention under low signal-to-noise ratio conditions. Journal of Experimental Psychology: General, 146, 651-671. doi:10.1037/xge0000284

Rangelov, D., Töllner, T., Müller, H. J., \& Zehetleitner, M. (2013). What are task-sets: A single, integrated representation or a collection of multiple control representations? Frontiers in Human Neuroscience, 7. 524. doi: $10.3389 /$ fnhum. 2013.00524

Rangelov, D., \& Zeki, S. (2014). Non-binding relationship between visual features. Frontiers in Human Neuroscience, 8, 749. doi:10. 3389/fnhum.2014.00749

Ratko-Dehnert, E. (2013). Distributional constraints on cognitive architecture: A statistical evaluation. (Doctoral dissertation). Retrieved from https://edoc.ub.uni-muenchen.de/15938
Reinhart, R. M. G., McClenahan, L. J., \& Woodman, G. F. (2016). Attention's accelerator. Psychological Science, 27, 790-798. doi: 10.1177/0956797616636416

Saiki, J. (2016). Location-unbound color-shape binding representations in visual working memory. Psychological Science, 27, 178-190. doi: $10.1177 / 0956797615616797$

Shomstein, S., \& Yantis, S. (2002). Object-based attention: Sensory modulation or priority setting? Perception \& Psychophysics, 64, 41-51. doi:10.3758/BF03194556

Soto, D., Heinke, D., Humphreys, G. W., \& Blanco, M. J. (2005). Early, involuntary top-down guidance of attention from working memory. Journal of Experimental Psychology: Human Perception and Performance, 31, 248-261. doi:10.1037/0096-1523.31.2.248

Soto, D., Hodsoll, J., Rotshtein, P., \& Humphreys, G. W. (2008). Automatic guidance of attention from working memory. Trends in Cognitive Sciences, 12, 342-348. doi:10.1016/j.tics.2008.05.007

Töllner, T., Gramann, K., Müller, H. J., Kiss, M., \& Eimer, M. (2008). Electrophysiological markers of visual dimension changes and response changes. Journal of Experimental Psychology: Human Perception and Performance, 34, 531-542.

Töllner, T., Zehetleitner, M., Krummenacher, J., \& Müller, H. J. (2011). Perceptual basis of redundancy gains in visual pop-out search. Journal of Cognitive Neuroscience, 23, 137-150.

Treisman, A., \& Souther, J. (1985). Search asymmetry: A diagnostic for preattentive processing of separable features. Journal of Experimental Psychology: General, 114, 285-310. doi:10.1037/ 0096-3445.114.3.285

Ulrich, R., Miller, J., \& Schröter, H. (2007). Testing the race model inequality: An algorithm and computer program. Behavior Research Methods, 39, 291-302. doi:10.3758/BF03193160

Watson, A. B., \& Pelli, D. G. (1983). QUEST: A Bayesian adaptive psychometric method. Perception \& Psychophysics, 33, 113-120. doi: $10.3758 / \mathrm{BF} 03202828$

Wolfe, J. M. (1998). Visual search. In H. Pashler (Ed.), Attention (pp. 1773). East Sussex: Psychology Press.

Wolfe, J. M. (2007). Guided search 4.0: Current progress with a model of visual search. In W. Gray (Ed.), Integrated models of cognitive systems (pp. 99-119). New York: Oxford University Press. 